\title{
Norethisterone-induced cholestasis: A case report
}

Safa moussaoui ${ }^{1}$, mehdi abdelwahed ${ }^{1}$, Nabil Ben Chaabene $^{1}$, Ahlem Bellalah $^{1}$, Najeh Ben Fadhel $^{1}$, Arwa Guediche ${ }^{1}$, Mejda Zakhama ${ }^{1}$, Ramzi Tabbabi ${ }^{1}$, Karim Aouam ${ }^{1}$, Zakhama Abdelfattah $^{1}$, Hichem Loghmari ${ }^{1}$, and Leila safer ${ }^{1}$

${ }^{1}$ Fattouma Bourguiba University Hospital of Monastir

December 21, 2021

\begin{abstract}
Case presentation: This case report concerns a 49-year-old woman who developed norethisterone drug -induced cholestasis, a progestin contraceptive, which is quite rare complication generally observed with estrogenic component of combined oral contraceptive pill.
\end{abstract}

\section{Hosted file}

CASE_REPORT_PROGESTERONE.docx available at https://authorea.com/users/452114/articles/550257norethisterone-induced-cholestasis-a-case-report 